OPEN ACCESS

Edited by:

Karl-Michael Schebesch, University of Regensburg, Germany

Reviewed by:

Jacopo Falco,

Fondazione IRCCS Istituto Neurologio Carlo Besta, Italy

Mehmet Osman Akçakaya, Istanbul Florence Nightingale Hospital, Turkey

*Correspondence: Maria Teresa Pedro maria-teresa.pedro@uni-ulm.de

Specialty section:

This article was submitted to Neuro-Oncology and Neurosurgical

Oncology,

a section of the journal Frontiers in Oncology

Received: 18 January 2021 Accepted: 12 February 2021 Published: 09 March 2021

Citation:

Pedro MT, Grübel N, Durner G, Pala A Wirtz CR and Koenig RW (2021) Intraoperative Sodium-Fluorescence Imaging in Peripheral Nerve Sheath Tumors (PNST) - A New Additional Promising Diagnostic Tool. Front. Oncol. 11:655392. doi: 10.3389/fonc.2021.655392

\section{Intraoperative Sodium-Fluorescence Imaging in Peripheral Nerve Sheath Tumors (PNST) - A New Additional Promising Diagnostic Tool}

\author{
Maria Teresa Pedro*, Nadja Grübel, Gregor Durner, Andrej Pala, Christian Rainer Wirtz \\ and Ralph Werner Koenig
}

Klinik für Neurochirurgie, Medizinische Fakultät, Universität Ulm, Ulm, Germany

Background: Through the development and implementation of specific fluorophore filters to microscopes in 2012, sodium fluorescein (SF) is currently experiencing a remarkable renaissance in neurosurgery. The present study examines its intraoperative application during surgical removal of peripheral nerve sheath tumors (PNST) and metastases.

Methods: This single-center study includes 10 cases of benign and malignant tumors as well as metastases of peripheral nerves (in total 11 PNST). Their surgical resections were all performed under microscope-based fluorescence with SF, which was administered intravenously (0.5-1.0 mg/kg body weight) during anesthesia induction. Microsurgical tumor removals were filmed and the collected data were retrospectively analyzed via ImageJ.

Results: Microsurgical tumor preparation was possible under the usage of fluorophore filter. In seven histological confirmed schwannoma ( $n=6$ patients) tissue differentiation between tumor mass and not involved fascicles was statistically significant for the colors green and red. Schwannoma maximum mean for green reached 254.7 pixel and 179.4 pixel for red, whereas passing healthy fascicles revealed a maximum mean for green 94.91 and for red 120.76 pixel. One case of neurofibroma achieved lower amount of pixel. Similar to schwannoma, the two MPNST cases showed a strong homogeneous fluorescence (max. mean green 215 pixel and red 124.51) involving the whole nerve segment. Subcutaneous tumor remnants were visualized and therefore resected. Via fascicular nerve biopsy a B-cell lymphoma of the tibial nerve could be detected. SF led to variable stain intensities in single fascicles. The resected fascicle revealed a max mean green of 100.54 pixel, whereas surrounding fascicles came up with max. mean green of 63.0 pixel.

Conclusions: Intraoperative SF visualization for PNST is feasible and of low risk. During resection of benign PNST, enhanced tissue differentiation between affected and not affected nerve segments is very useful. Tumor remnants can be detected safely and effectively. Its application during resection of malignant PNST is limited. Due to the infiltrative nature of those tumors, intraneural tissue differentiation is not possible. 
"Fluorescence-guided" biopsy can be regarded as an additional advantage in PNST surgery. Due to the encouraging experience in our institution SF was established as standard visualization tool in PNST surgery.

Keywords: schwannoma, neurofibroma, malignant peripheral nerve sheat tumor, lymphoma, biopsy, sodium-fluorescein

\section{INTRODUCTION}

\section{Sodium-Fluorescein}

Although an American neurosurgeon was the first one to describe the application of Sodium fluorescein (SF) in brain surgery in 1948 (1), this fluorphore medium fell into oblivion.

Only in 2003 and 2010 Shinoda and Okuda published their results in brain tumor surgeries under the usage of $\mathrm{SF}(2,3)$. Their patients received intravenously a dosage of $20 \mathrm{mg} / \mathrm{kg}$ bodyweight which led to a distinct tumor mass fluorescence under white light.

However, since 2012 through the development and implementation of specific fluorophore filters to microscopes (e.g., YELLOW $560 \mathrm{~nm}$ filter; Zeiss Meditec, Oberkochen, Germany) its dosage has been significantly reduced to $2-5 \mathrm{mg} / \mathrm{kg}$ bodyweight. By this means, until today SF has experienced a tremendous renaissance in neurosurgery (4-8).

\section{Peripheral Nerve Sheath Tumors}

Since 2013 the WHO classification has no longer assigned peripheral nerve sheath tumors to tumors of the central nervous system, but to tumors of the soft tissues and bones (9). Schwannoma and neurofibroma each make up about $5 \%$ of the soft tissue tumors (10), both rare entities are considered as benign. Their surgical enucleation should not lead to neurological deterioration. But depending on location, size and tissue consistency its surgical preparation may be demanding.

Malignant peripheral nerve sheath tumors (MPNST) are aggressive infiltrative growing tumors leading to a remarkable quickly emerging neurological impairment and resting pain. Until now the only effective therapy is surgical resection with wide negative margins (11). While post-operative radiation is recommended, the efficacy of chemotherapy remains unclear $(11,12)$.
The present study examines our experience and benefits of intraoperative SF application during surgical removal of different peripheral nerve sheath tumors (PNST) and metastases.

\section{METHODS}

\section{Study Group, Dosage, and Technical Equipment}

This present single-center study includes 10 cases of benign and malignant tumors as well as metastases of peripheral nerves (in total 11 PNST). Patients over $\geq 18$ years of age and with suspicion of PNST in MRI were included, whereas patients with history of renal insufficiency or SF intolerance were excluded. Their surgical PNST resections, respectively, biopsies were all performed by one surgeon under microscope-based fluorescence with SF (YELLWO $560 \mathrm{~nm}$ filter, Zeiss Meditec, Oberkochen, Germany) between August 2017 and September 2020 (Table 1). SF (Fluorescein Alcon 10\%, Freiburg, Germany) was administered intravenously $(0.5-1.0 \mathrm{mg} / \mathrm{kg}$ body weight) directly during anesthesia induction.

Since the application of SF is still restricted to ophthalmologic indications in Germany, the neurosurgical usage was off- label. Therefore, informed written consents were obtained from all patients emphasizing its intraoperative use. As individualized treatment, this study is in accordance with the ethical principles that are reflected in the Declaration of Helsinki.

\section{Image Evaluation}

Microsurgical preparation was filmed and the collected data were retrospectively analyzed via ImageJ, an open source Java image processing platform (Version 1.51, National Institutes of Health, Bethesda, Maryland, USA) (13). Therefore, regions of interest

TABLE 1 | Study group overview.

\begin{tabular}{|c|c|c|c|c|c|}
\hline Patient & Age (years); Sex & Affected nerve & Histology & Resection (complete/biopsy) & Surgeon's opinion \\
\hline 1 & 24 yrs; female & Median nerve & Schwannoma & Complete & Helpful \\
\hline 2 & 45 yrs; female & Sural nerve & Schwannoma & Complete & Helpful \\
\hline 3 & 50 yrs; male & Tibial nerve & Schwannoma & Complete & Helpful \\
\hline 4 & 37 yrs; female & Median nerve & Schwannoma & Complete & Helpful \\
\hline 5 & 48 yrs; male & Peroneal nerve & 2x schwannoma & Complete & Helpful \\
\hline 6 & 41 yrs; female & Peroneal nerve & Schwannoma & Complete & Helpful \\
\hline 7 & 45 yrs; male & Median nerve & Neurofibroma & Complete & Helpful \\
\hline 8 Illustrative case & 77 yrs; female & Cutanaeus nerve paraspinal & MPNST & Complete & Very helpful \\
\hline 9 Illustrative case & 55 yrs; male & Ulnar nerve & MPNST & Biopsy & Not helpful \\
\hline 10 & 71 yrs; male & Tibial nerve & B-cell lymphoma & Biopsy & Helpful \\
\hline
\end{tabular}




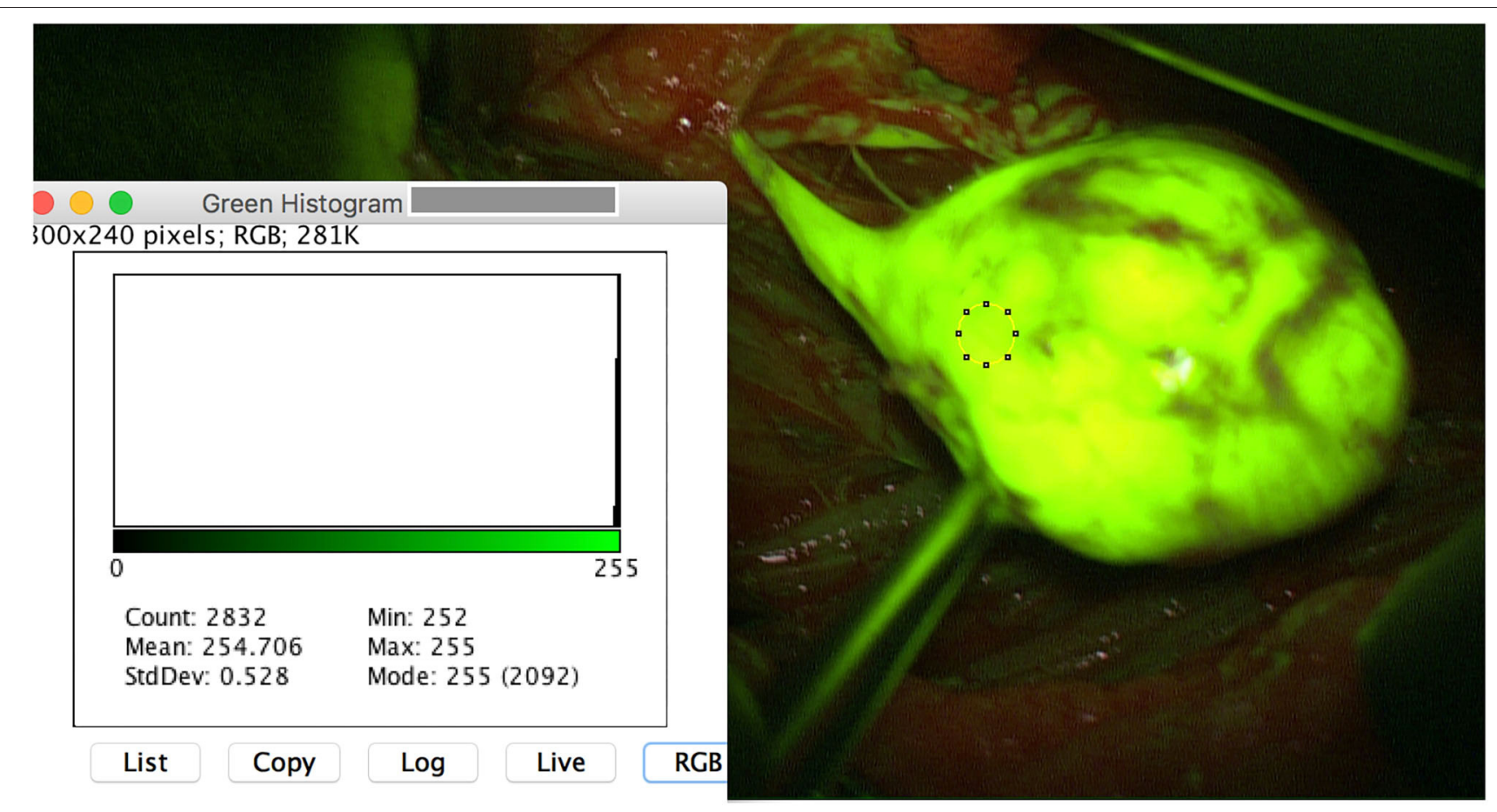

FIGURE 1 | Intraoperative image of a schwannoma of the left tibial nerve after opening of the epineurium. The region of interest (ROI) is set on the tumor mass. Image $\mathrm{J}$ reveals a mean $\max$ for the color green of 255 pixel with a standard deviation of 0.528 . Note that the originating fascicle reveals a high yellowish stain.

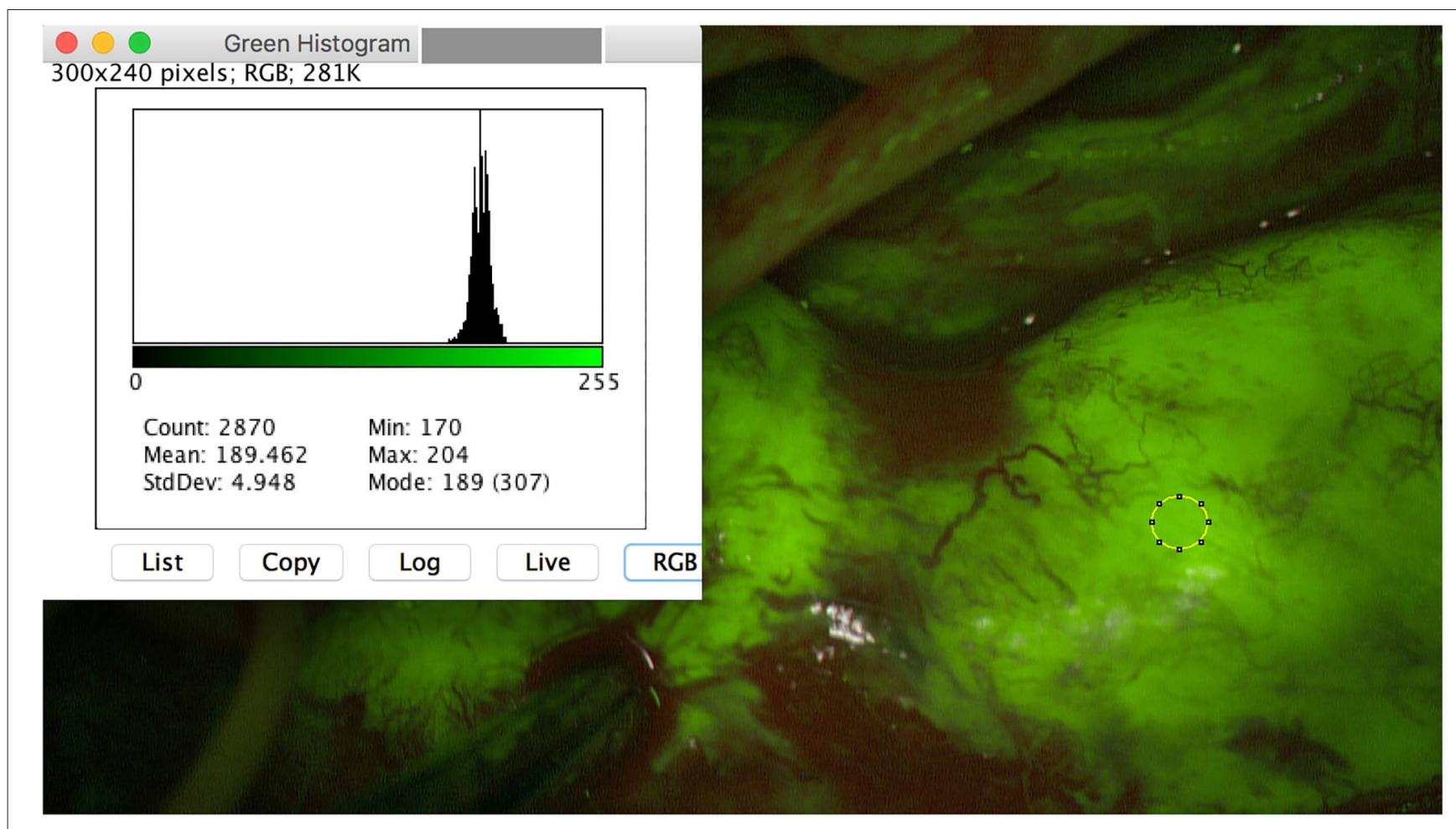

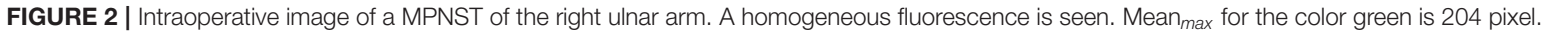


(ROI) were set on the tumor mass and on inconspicuous nerve segments. For each ROI the quantity of the complementary colors red and green were evaluated.

\section{Data Analysis}

For statistical analysis, SAS software version 9.4 (SAS Institute Inc., Cary, North Carolina, USA) was applied. First of all, frequencies, mean values as well as position and dispersion measures were calculated. For the latter, the representation was done with box plot diagrams. The test for normal distribution was carried out with the Shapiro-Wilk test. The non-parametric Mann-Whitney-U test was used to compare interval-scaled variables and the central tendency. The tests were carried out on both sides. $P<0.05$ ( $\alpha=0.05)$ were rated as statistically significant with an error probability of five percent.

\section{RESULTS}

\section{General Results}

The present cohort consists of each 5 male and female patients ranging in age from 24 to 77 years (mean age, 49 years). In total 11 PNST were histologically examined ( $7 \mathrm{x}$ schwannoma, $1 \mathrm{x}$ neurofibroma, 2x MPNST and 1x B-cell lymphoma). Complete tumor resection was performed in 8 patients, whereas 2 cases underwent fascicular biopsy (1x MPNST and 1x B-cell lymphoma). In 9 out of 10 cases (90\%) the application of SF during tumor preparation was described as helpful by the surgeon (Table 1). No adverse events occurred under the applied dosage of $0.5-1.0 \mathrm{mg} / \mathrm{kg}$ bodyweight.

\section{Sodium-Fluorescein Results Correlating to Histopathology}

Schwannoma reached a max. mean green of 254.7 pixel and a max. mean $_{\text {red }}$ of 179.4 pixel (Figure 1). In contrast, the passing healthy fascicles revealed a max. mean green $_{\text {of }} 94.91$ pixel and a max. mean $_{\text {red }}$ of 120.76 pixel. The one neurofibroma case achieved a lower amount of fluorescence intensity. Its tumor mass max. mean $n_{\text {green }}$ reached 140.9 pixel and max. mean $n_{\text {red }} 76.2$ pixel. Its not affected fascicles 88.5 (max. mean $_{\text {green }}$ ) and 66.6 (max. mean red ) pixel.

Similar to schwannoma, the MPNST achieved high SF intensities. One of both MPNST patients revealed an untypical MPNST anamnesis and tumor infestation, so that both cases are highlighted additionally as illustrative cases. MPNST max. mean $_{\text {green }}$ was 215 and max. mean red $_{\text {was }} 124.5$ pixel. MPNST fluorescence was homogenous strong involving the whole nerve segment (Figure 2). Whereas, the surrounding subcutaneous tissue achieved lower values of 81.4 ( $\max$. mean $_{\text {green }}$ ) and 73.4 $\left(\max\right.$. mean $\left._{\text {red }}\right)$. One patient suffering of a B-cell lymphoma in

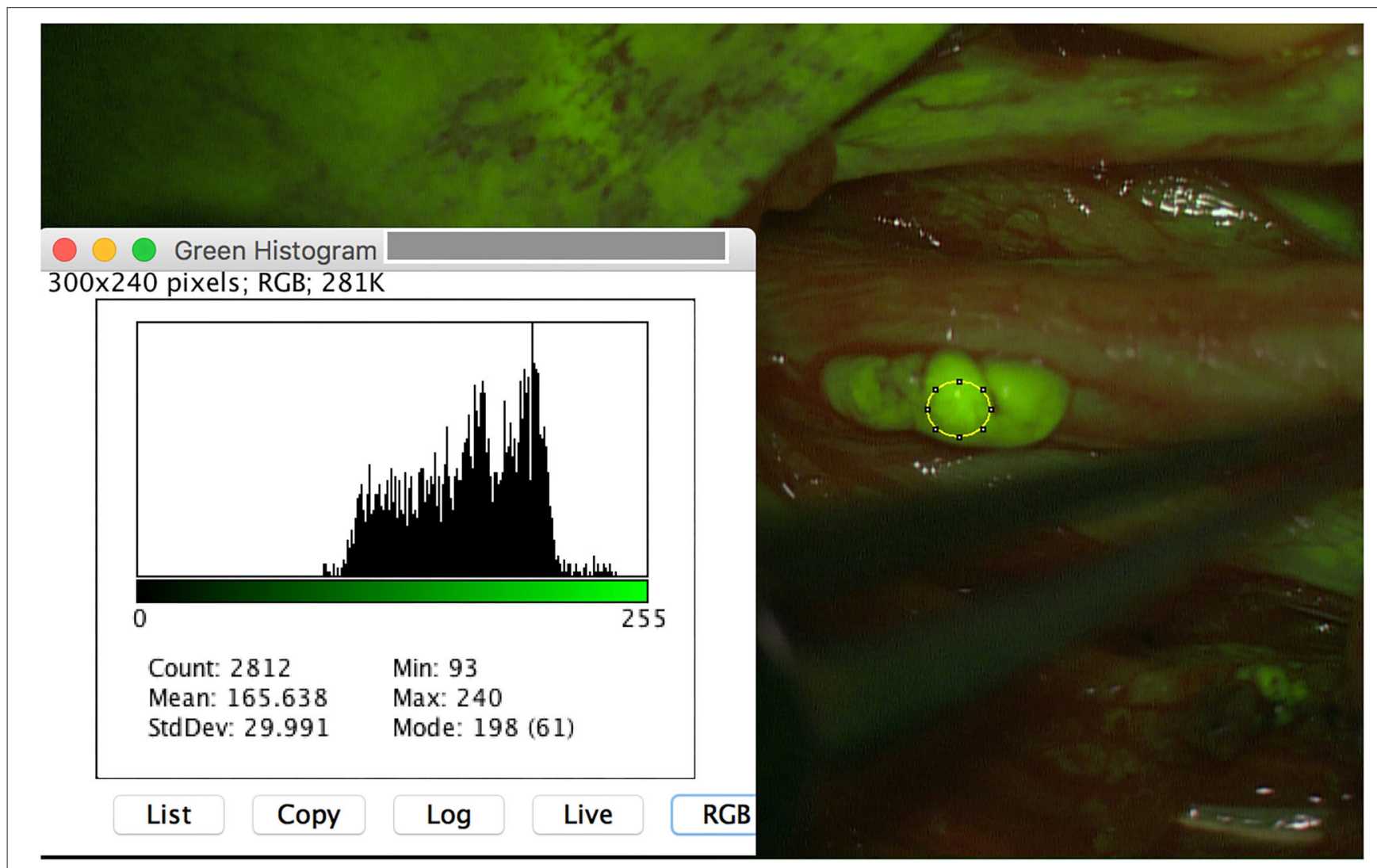

FIGURE 3 | A small schwannoma $(3 \times 2 \mathrm{~mm})$ of the left peroneal nerve is additionally detected with SF, achieving similar results of mean max $_{\text {as }}$ the other schwannoma (240 pixel). 


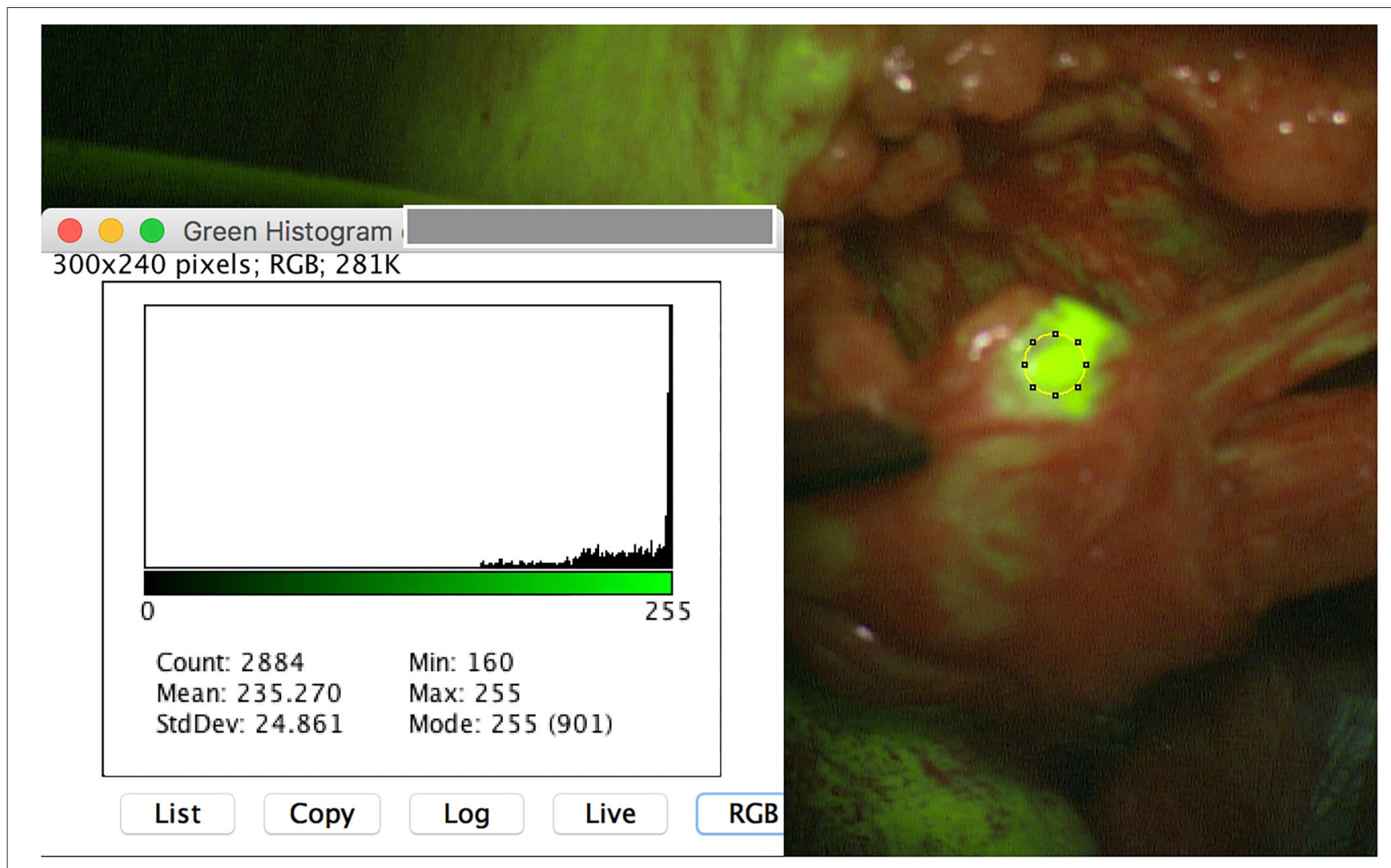

FIGURE 4 | Example of visibility of fluorescent schwannoma remnant of right peroneal nerve.

TABLE 2 | Green and red mean differences between healthy tissue and affected tissue.

\begin{tabular}{lccc}
\hline & \multicolumn{2}{c}{ Mean \pm SD (Median) Min-Max } & \\
\cline { 2 - 3 } $\begin{array}{l}\text { Complementary } \\
\text { colors }\end{array}$ & $\begin{array}{c}\text { Healthy tissue } \\
(\boldsymbol{n}=\mathbf{1 1})\end{array}$ & $\begin{array}{c}\text { Affected issue } \\
(\boldsymbol{n}=\mathbf{1 1})\end{array}$ & $\boldsymbol{p}$-Value \\
\hline Green mean & $\begin{array}{c}83.36 \pm 24.04 \\
(78.00)\end{array}$ & $\begin{array}{c}198.48 \pm 49.97 \\
(189.46)\end{array}$ & \multirow{2}{*}{0.0001} \\
value & $54.17-145.54$ & $100.54-254.71$ & \\
Red mean & $78.52 \pm 20.13$ & $120.16 \pm 37.67$ & 0.0071 \\
value & $(83.58)$ & $(111.94)$ & \\
& $44.02-120.76$ & $57.59-179.41$ & \\
\hline
\end{tabular}

Counts are in pixel, $p<0.05$ is determined as statistically significant.

his medical history for 3 years, reported of a slowly progressing weakness of his right foot flexion. Since 2017 after chemotherapy he was already suffering a severe polyneuropathy. MRI showed a longitudinal positive contrast enhancement of a thickened tibial nerve. During tibial nerve biopsy, SF led to variable stain intensities in single fascicles. The resected fascicle revealed a max. mean $_{\text {green }}$ of 100.54 and max. mean $n_{\text {red }}$ of 57.6 pixel. Surrounding fascicles came up with max. mean green values of 63 and max. mean $_{\text {red }}$ of 44 pixel.
Besides all these results, surgeon described the SF application as useful, since even very small PNST (for instance schwannoma $3 \times 2 \mathrm{~mm}$ ) were safely intraoperatively detected (Figure 3). Moreover, tumor remnants were securely depicted and resected (Figure 4).

In summary, in this present study group involving different PNST, tissue differentiation via SF was found to be statistically significant for the colors green $(p=0.0001)$ and red $(p=0.0071)$ (Table 2 and Figures 5A,B). Therefore, it is considered as a helpful intraoperative tool.

\section{DISCUSSION}

\section{Sodium-Fluorescein in Brain Surgery}

The rediscovery and renaissance of SF in neurosurgery, started with its application during brain tumor surgery in the early 2000 (2). Indeed, currently many neurosurgical publications deal with SF. Through technical evolution of specific fluorescence filters and their implementation to microscopes in 2012, SFvisualization was significantly improved whereas its applied dosage was reduced. Subsequently in 2013 Schebesch et al. (14) published their first experience in sodium fluorescein-guided resections of malignant brain tumors. They found SF to be safe and feasible (14). At the same time, similar results were reported 


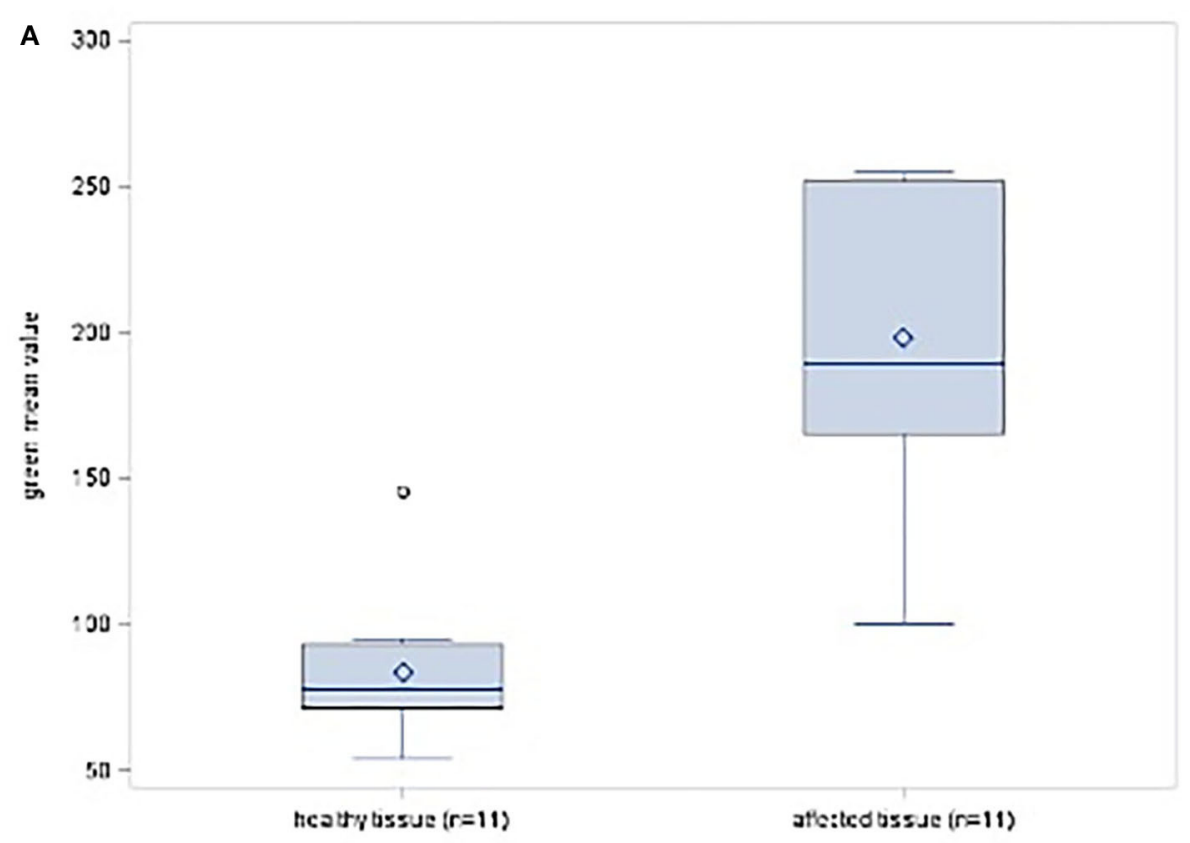

B

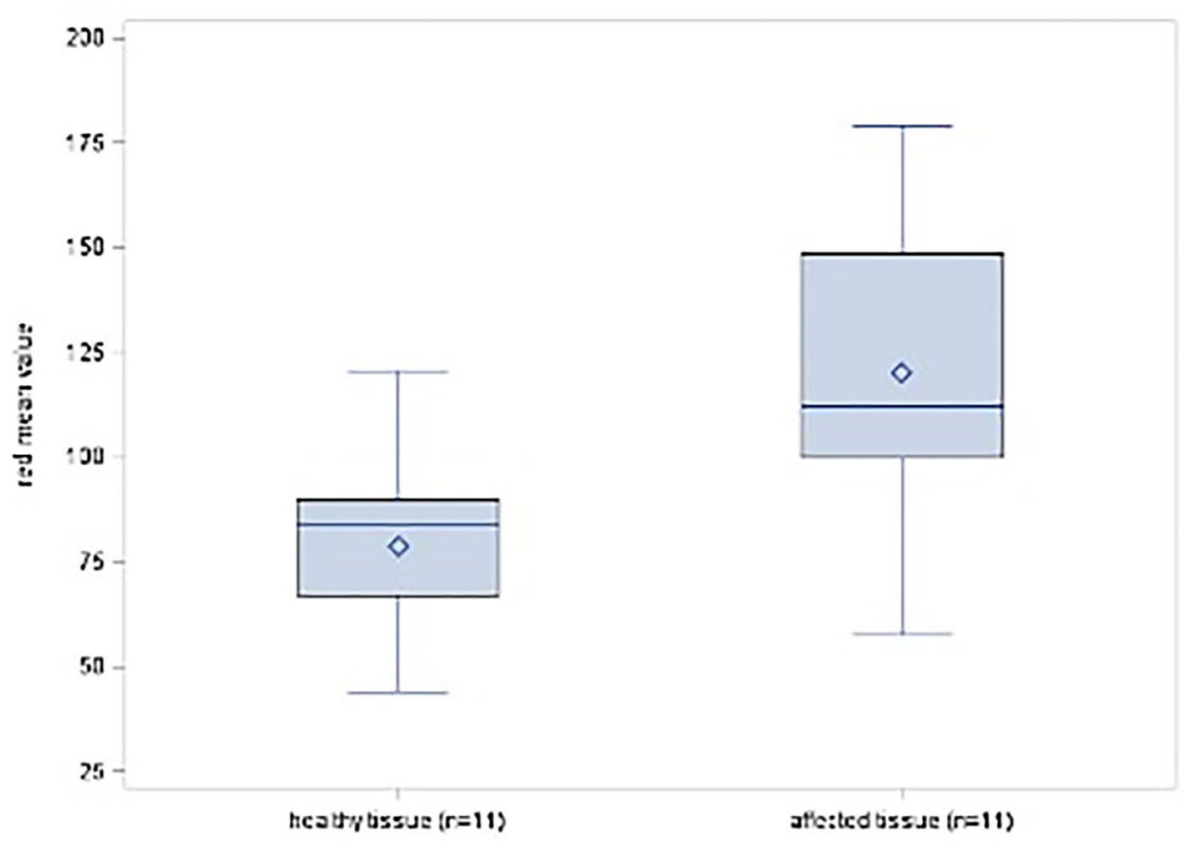

FIGURE 5 | (A) Box-plot diagram showing the distribution scores for the color green in healthy and affected tissues. (B) Box-plot diagram showing the distribution scores for the color red in healthy and affected tissues.

by an American group, they even extended SF's application to neurovascular malformations (7). Currently, SF is frequently used in surgeries of high-grade, low-grade gliomas, as well as meningioma or brain metastases $(5,6,8,15)$. Furthermore, SF is meanwhile applied in neuropediatrics $(4,16,17)$. Due to this lower dosage of $2.0-5.0 \mathrm{mg} / \mathrm{kg}$ bodyweight no adverse events were reported in all those studies.
Nonetheless, the usage of SF in brain surgery has also been criticized by other study groups. In contrast to 5-aminolevulinic acid, SF reveals no glioblastoma cell-specific accumulation (18). After developing several in vitro and in vivo study models to investigate SF's biodistribution, Diaz et al. (18) came to the conclusion that SF accumulates in the extracellular space most likely due to the blood-brain-barrier dysfunction, similar to 
gadolinium contrast enhancement in MRI. Meanwhile, however, a recent study describes via SF a yellowish intraoperative fluorescence during resection of non-gadolinium positive brain tumors. Tumor detection and identification of its margins were possible in those gliomas. As common feature, they were all positive on F-fluoroethyltyrosine positron emission tomography (19).

In their most recent study, Bömers et al. (20) focused on surgeon reported usability of SF during glioblastoma surgery. Similar to the study results of Erdman et al. (16) and de Laurentis et al. (17) a high user satisfaction was observed (20). The identification of tumor location, margins and the secure microsurgical tumor resection under the SF filter was highlighted in all above-mentioned studies. Those results correlate well with our findings during PNST surgery.

\section{Sodium-Fluorescein in Peripheral Nerve Sheath Tumors (PNST)}

Meanwhile, the application of SF is becoming a common tool for intraoperative tumor visualization, including intraneural ganglion cysts formations, in peripheral nerve surgery (2124). According to our present results, it is a safe and useful method, which helps to define clearly tumor borders and hereby achieve gross total resection. Additionally, we were able to quantify these results using an imaging process platform (imageJ) and describe the different spectral ranges within different PNST and healthy tissue. Furthermore, in 10 out of 11 PNST resections, respectively, biopsies surgeon found SF to be helpful in this present analysis. As described in 2019, an application of low dose SF (0.5-1.0 mg/kg bodyweight) in an early stage (i.e., anesthesia induction), leads to a tissue differentiation between schwannoma and en passant fascicles. At the same time the standard principles of PNST microsurgical resection (i.e., direct motoric stimulation, EMG, NAPs, longitudinal dissection) have to be respected to preserve neurological function (21). Similar results were found by an Italian group in 2019. Vetrano et al. (22) examined 25 cases of PNST during surgery under SF. An optimal distinction between tumor and surrounding nerves was observed in 13 out of 14 schwannoma and in all 8 neurofibroma (22). Moreover, they emphasize the fact, that tumor remnants were not seen in 7 cases under white-light, but under SF (22). In contrast to those findings, a French group, examined 5 cases of schwannoma under the same circumstances. They did not see a benefit in between the tumor visualization under white-light and SF (25). Kalamarides stresses the need of intraoperative fascicular mapping and monitoring as stand-alone modality (25). All schwannoma were resected en-bloc, so that the need to depict possible tumor remnants did not arise. As seen in 2019 and in these present results, it is not always possible to detach schwannoma tissue from the healthy fascicles in toto, piecemeal technique has to be applied in some cases. Since a grosstotal resection should be achieved, it is definitely reasonable to implement SF during schwannoma, respectively, neurofibroma resection $(21,22,26)$.

MPNST are aggressive soft tissue sarcoma that account for $\sim 5-10 \%$ of all cases (27). They mainly arise de novo in peripheral nerves or from neurofibroma (28).

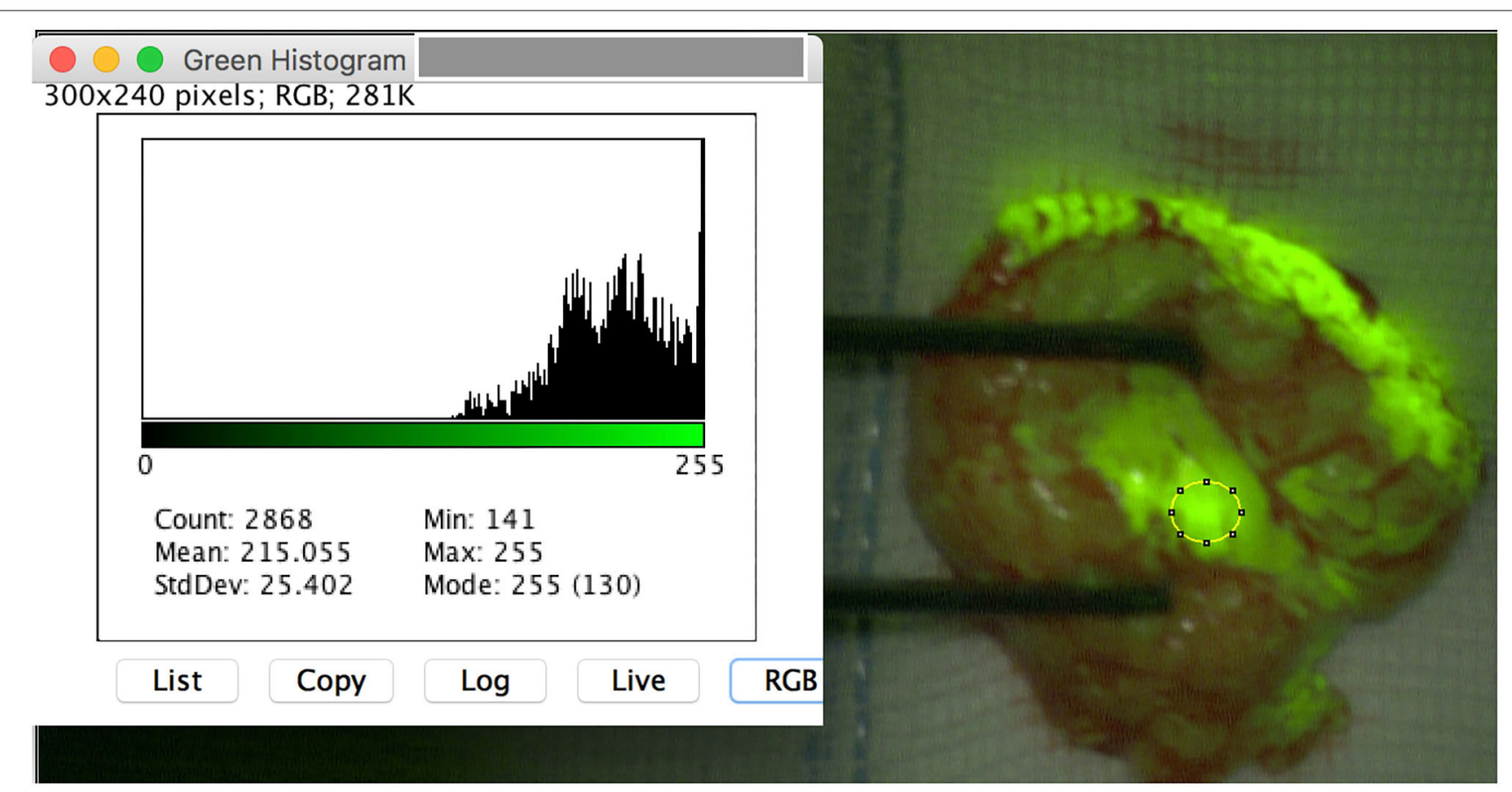

FIGURE 6 | Subcutaneous resected tissue of MPNST of a paraspinal nerve (pat. $\mathrm{n}^{\circ}$ 8). Yellowish tumor remnants became visible. 
Until now the only effective therapy and positive predictor of survival is surgical resection with wide negative margins $(11,12)$. While post-operative radiation is recommended, the efficacy of chemotherapy is controversially discussed $(11,12)$. As illustrated below, two MPNST patients were operated under the usage of SF. In one case, the whole ulnar nerve achieved a strong homogeneous yellowish stain, so that an intraneural tissue differentiation was not seen under the SF filter. Its usage was considered as not helpful during biopsy. The other case, a MPNST manifestation most likely out of a paraspinal cervical nerve, was operated several times, since under white-light microscopy and frozen section analysis a gross total resection of the surrounding tissue was not achieved. During the third surgery, SF revealed fluorescence of subcutaneous tissue, so that by this means, a complete resection became possible. After radiotherapy the patient reveals no recurrence or metastases until now.

Despite MRI, ultrasound and electrophysiology, there are some cases of peripheral nerve lesions, that need fascicular biopsy for further diagnosis in order to determine the proper therapy $(23,29)$. Those lesions may look inconspicuous in white-light microscopy, so that the usage of SF has been additionally examined in those cases. As described in 2020, the intraoperative application of SF helps to visualize the most affected fascicles and to determine a target fascicle biopsy (23). In this study group one patient, suffering of a B-cell lymphoma, developed neurological deficits of the tibial nerve. MRI showed a longitudinal nerve enlargement and contrast enhancement.
Oncologists set the indication for nerve biopsy. During surgery, different intensities of fluorescence were seen, after motoric mapping of the fascicles, surgeon decided to resect one strong fluorescent fascicle. Histopathology confirmed the manifestation of a B-cell lymphoma.

In summary, SF, applied in low dose and during anesthesia induction, is meaningful and easily feasible during PNST removals or biopsies. No adverse events occurred.

In future, direct implementation of an image processing platform tool into the microscope could additionally enhance the value of this technique. By this means, surgeons would immediately receive mean values of relevant regions of interest in real time. Thereby, differentiated intraoperative decision making would be further simplified and improved.

\section{Illustrative Cases}

Patient no. 8 (Table 1), a 77 years old female, suffered of a walnut-sized tumorous subcutaneous nuchal formation. She had no pain or neurological deficits. By suspicion of an atheroma she underwent outpatient surgery. Histopathology revealed a MPNST, therefore, she was timely admitted to our clinical center. Up to this point of time, there were no preexisting neurological diseases, no signs of neurofibromatosis or significant comorbidities.

She was conducted to MRI and FDG-PET. Imaging depicted residual tumor mass and high accumulation of the surrounding tissue. Metastases were not detected. The second tumor

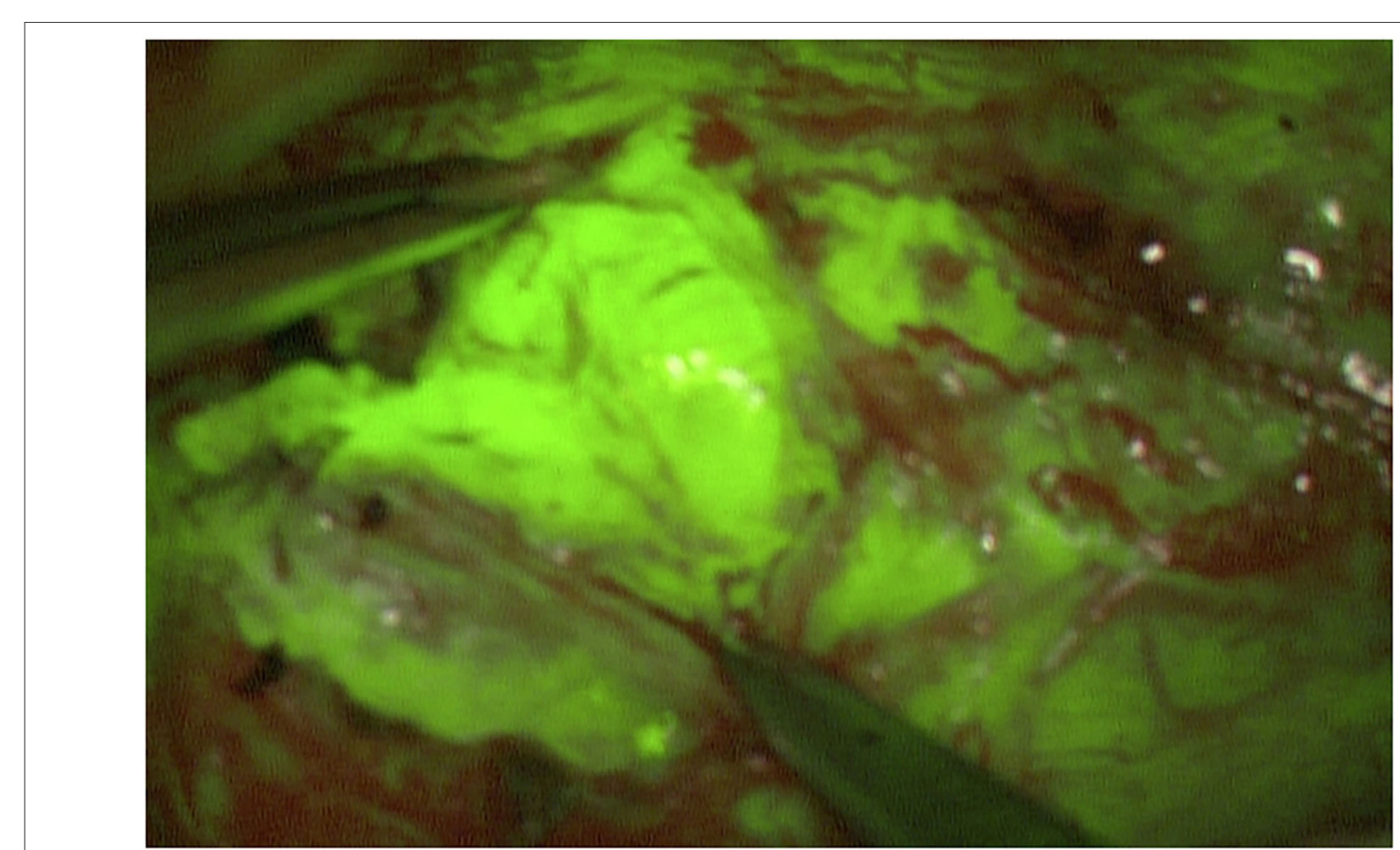

FIGURE 7 | After opening of the epineurium of the ulnare nerve, the complete MPNST tissue reveals a strong homogeneous fluorescence. An intraneural tissue differentiation is not possible via SF (pat. $n^{\circ} 9$ ). 
resection was performed under white light. However, tumor free margins were not achieved according to the final histopathology results. As consequence, SF was applied during the third tumor resection. Subcutaneous tissue showed different degrees of fluorescence (Figure 6). Strong fluorescent areas were all resected. A complete tumor removal was finally confirmed by histopathology. A subsequent radiation followed. Until now, 18 months later, patient reveals no recurrence or metastases in the diagnostic follow-up.

Patient no. 9 (Table 1), a 55 years old male, suffered of slow progressing paralysis of his right hand for years. He described an hypesthesia of his ring and small finger, so that on suspicion of a Loge de Guyon entrapment syndrome, he underwent decompression of his right ulnar nerve. Three years later, pain and functional loss increased dramatically. MRI and ultrasound depicted a thickened, strong enhancing ulnar nerve along the complete upper arm. The patient revealed no signs of neurofibromatosis or schwannomatosis. There were no preexisting diseases. Fascicular biopsy was performed under SF and direct motoric stimulation. After epineurotomy, all fascicles of the ulnar nerve showed a homogenous strong fluorescence. An intraneural tissue differentiation was not recognizable (Figure 7).

Histopathology resulted in MPNST. In accordance to the local tumor board, the right arm was amputated. Metastases were not detected at this point. Seven months later, the patient developed pulmonary metastases on both lungs. Besides surgical resection, he died 2 months later.

\section{CONCLUSION}

During the resection of benign PNST, tissue differentiation between affected and not affected nerve segments became

\section{REFERENCES}

1. Moore GE, Peyton WT, French LA, Walker WW. The clinical use of fluorescein in neurosurgery; the localization of brain tumors. J Neurosurg. (1948) 5:392-8. doi: 10.3171/jns.1948.5.4.0392

2. Shinoda J, Yano H, Yoshimura S, Okumura A, Kaku Y, Iwama T, et al. Fluorescence-guided resection of glioblastoma multiforme by using highdosage fluorescein sodium. Technical note. J Neursurg. (2003) 99:597603. doi: 10.3171/jns.2003.99.3.0597

3. Okuda T, Kataoka K, Yabuuchi T, Yugami H, Kato A. Fluorescence-guided surgery of metastatic brain tumors using fluorescein sodium. J Clin Neurosci. (2010) 17:118-21. doi: 10.1016/j.jocn.2009.06.033

4. Göker B, Kiris T. Sodium fluorescein-guided brain tumor surgery under the Yellow-560-nm surgical microscope filter in pediatric age group: feasibility and preliminary results. Childs Nerv Syst. (2019) 35:42935. doi: 10.1007/s00381-018-04037-4

5. Xiao SY, Zhang J, Zhu ZQ, Li YP, Zhong WY, Chen JB, et al. Application of fluorescein sodium in breast cancer brain-metastasis surgery. Cancer Manag Res. (2018) 10:4325-31. doi: 10.2147/CMAR.S176504

6. Schebesch KM, Hoehne J, Hohenberger C, Proescholdt M, Riemenschneider MJ, Wendl C, et al. Fluorescein sodium-guided resection of cerebral metastases- experience with the first 30 patients. Acta Neurochir. (2015) 157:899-904. doi: 10.1007/s00701-015-2395-7

7. Rey-Dios R, Cohen-Gadol AA. Technical principles and neurosurgical applications of fluorescein fluorescence using a visible using SF. Tumor remnants were securely detected. This intraoperative visualization method seems to be a helpful tool for surgeons. In contrast, the usage in malignant PNST was limited. In those cases, an intraneural tissue differentiation was not possible, only the extraneural surrounding tissues revealed a far lower fluorescence than the MPNST. Concerning the sciatic infestation of lymphoma, SF was helpful in identifying affected nerve fascicles.

The intraoperative application of SF in PNST surgery has been established as standard visualization tool in the present clinical institution.

\section{DATA AVAILABILITY STATEMENT}

The raw data supporting the conclusions of this article will be made available by the authors, without undue reservation.

\section{ETHICS STATEMENT}

Ethical review and approval was not required for the study on human participants in accordance with the local legislation and institutional requirements. The patients/participants provided their written informed consent to participate in this study.

\section{AUTHOR CONTRIBUTIONS}

MP principal investigator and data analysis. RK conception and ImageJ evaluation. CW study design and counselor. AP data analysis and study design. GD data analysis. NG ImageJ evaluation and study design. All authors contributed to the article and approved the submitted version. microscope-integrated fluorescence module. Acta Neurochir. (2013) 155:701-6. doi: 10.1007/s00701-013-1635-y

8. Acerbi F, Broggi M, Eoli M, Anghileri E, Cuppini L, Pollo B, et al. Fluoresceinguided surgery for grade IV gliomas with a dedicated filter on the surgical microscope: preliminary results in 12 cases. Acta Neurochir. (2013) 155:127786. doi: 10.1007/s00701-013-1734-9

9. Fletcher CDM, Bridge JA, Hogendoorn P, Mertens F. WHO Classification of Tumours of Soft Tissue and Bone. 4th ed. Lyon: IARC Press (2013)

10. Kransdorf MJ. Benign soft-tissue tumors in a large referral population: distribution of specific diagnoses by age, sex, and location. AJR. (1995) 164:395-402. doi: 10.2214/ajr.164.2.7839977

11. Reilly KM, Kim A, Blakely J, Ferner RE, Gutmann DH, Legius E, et al. Neurofibromatosis type 1- associated MPNST state of the science: outlining a research agenda for the future. J Natl Cancer Inst. (2017) 109:djx124. doi: 10.1093/jnci/djx124

12. James AW, Shurell E, Singh A, Dry SM, Eilber FC. Malignant peripheral nerve sheath tumor. Surg Oncol Clin N Am. (2016) 25:789-802. doi: 10.1016/j.soc.2016.05.009

13. Schneider CA, Rasband WS, Eliceiri KW. NIH image to ImageJ: 25 years of image analysis. Nat Methods. (2012) 9:671-5. doi: 10.1038/nmeth.2089

14. Schebesch KM, Proescholdt M, Hoehne J, Hohenberger C, Hansen E, Riemenschneider $\mathrm{MJ}$, et al. Sodium fluorescein-guided resection under the YELLOW $560 \mathrm{~nm}$ surgical microscope filter in malignant brain tumor surgery-a feasibility study. Acta Neurochir. (2013) 155:6939. doi: $10.1007 / \mathrm{s} 00701-013-1643-y$ 
15. Schebesch KM, Brawanski A, Hohenberger C, Hohne J. Fluorescein sodium-guided surgery of malignant brain tumors: history, current concepts, and future project. Turk Neurosurg. (2016) 26:185-94. doi: 10.5137/1019-5149.JTN.16952-16.0

16. Erdman CM, Christie C, Iqbal MO, Mazzola CA, Tomycz L. The utilization of sodium fluorescein in pediatric brain stem gliomas: a case report and review of the literature. Childs Nerv Syst. (2020). doi: 10.1007/s00381-020-04857-3. [Epub ahead of print].

17. De Laurentis C, Hoehne J, Cavallo C, Restelli F, Falco J, Broggi M, et al. The impact of fluorescein-guided technique in the surgical removal of CNS tumors in a pediatric population: results from a multicentric observational study. $J$ Neurosurg Sci. (2019) 63:679-87. doi: 10.23736/S0390-5616.19.04601-0

18. Diaz RJ, Dios RR, Hattab EM, Burell K, Rakopoulos P, Sabha N, et al. Study of the biodistribution of fluorescein in glioma-infiltrated mouse brain and histopathological correlation of intraoperative findings in high-grade gliomas resected under fluorescein fluorescence guidance. J Neurosurg. (2015) 122:1360-9. doi: 10.3171/2015.2.JNS132507

19. Schebesch KM, Brawanski A, Doenitz C, Rosengarth K, Proescholdt M, Riemenschneider MJ, et al. Fluorescence-guidance in nonGadoliniumenhancing, but FET-PET positive gliomas. Clin Neurol Neurosurg. (2018) 172:177-82. doi: 10.1016/j.clineuro.2018.07.011

20. Bömers JP, Danielsen ME, Schulz MK, Halle B, Kristensen BW, Sørensen MD, et al. Sodium fluorescein shows high surgeonreported usability in glioblastoma surgery. Surgeon. (2020) 18:344-8. doi: 10.1016/j.surge.2020.01.003

21. Pedro MT, Eissler A, Schmidberger J, Kratzer W, Wirtz CR, Antoniadis G, et al. Sodium fluorescein-guided surgery in peripheral nerve sheath tumors: first experience in 10 cases of schwannoma. World Neurosurg. (2019) 17:e51321. doi: 10.1016/j.wneu.2019.01.010

22. Vetrano IG, Acerbi F, Falco J, Devigili G, Rinaldo GM, Prada F, et al. Fluorescein-guided removal of nerve sheath tumors: a preliminary analysis of 20 cases. J Neurosurg. (2019) 6:1-10. doi: 10.3171/2019.9.JNS19970

23. Pedro MT, Eissler A, Scheuerle A, Schmidberger J, Kratzer W, Wirtz CR, et al. Sodium fluorescein as intraoperative visualization tool during peripheral nerve biopsies. World Neurosurg. (2020) 133:e513-21. doi: 10.1016/j.wneu.2019.09.081

24. Stone JJ, Graffeo CS, de Ruiter GCW, Rock MG, Spinner RJ. Intraoperative intravenous fluorescein as an adjunct during surgery for peroneal intraneural ganglion cysts. Acta Neurochir. (2018) 160:651-4. doi: 10.1007/s00701-018-3477-0

25. Kalamarides M, Bernat I, Peyre M. Extracapsular dissection in peripheral nerve schwannoma surgery using bright light and fluorescein sodium visualization: case series. Acta Neurochir. (2019) 161:2447-52. doi: 10.1007/s00701-019-04071-4

26. Vetrano IG, Nazzi V, Acerbi F. What is the advantage of using sodium fluorescein during resection of peripheral nerve tumors? Acta Neurochir. (2020) 162:1157. doi: 10.1007/s00701-019-04211-w

27. Fuchs B, Spinner RJ, Rock MG. Malignant peripehral nerve sheath tumors: an update. J Surg Orthop Adv. (2005) 14:168-74.

28. Wong WW, Hirose T, Scheithauer BW, Schild SE, Gunderson LL. Malignant peripheral nerve sheath tumor: analysis of treatment outcome. Int J Radiat Oncol Biol Phys. (1998) 42:351-60. doi: 10.1016/S0360-3016(98)0 0223-5

29. Brand C, Pala A, Scheuerle A, Scheglmann K, König R, Kratzer W, et al. Neurolymphomatosis: two case reports. Nervenarzt. (2017) 89:7014. doi: 10.1007/s00115-017-0460-6

Conflict of Interest: The authors declare that the research was conducted in the absence of any commercial or financial relationships that could be construed as a potential conflict of interest.

Copyright (c) 2021 Pedro, Grübel, Durner, Pala, Wirtz and Koenig. This is an openaccess article distributed under the terms of the Creative Commons Attribution License (CC BY). The use, distribution or reproduction in other forums is permitted, provided the original author(s) and the copyright owner(s) are credited and that the original publication in this journal is cited, in accordance with accepted academic practice. No use, distribution or reproduction is permitted which does not comply with these terms. 